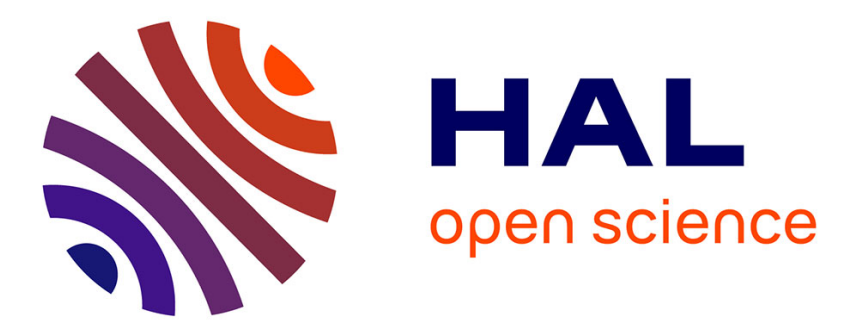

\title{
Cloud-based spectrum sensing system: First field results
}

\author{
Sylvain Azarian, Loig Godard, Merouane Debbah
}

\section{To cite this version:}

Sylvain Azarian, Loig Godard, Merouane Debbah. Cloud-based spectrum sensing system: First field results. 2014 1st International Workshop on Cognitive Cellular Systems (CCS 2014), Sep 2014, Rhine River, Germany. 10.1109/CCS.2014.6933809 . hal-01098822

\section{HAL Id: hal-01098822 \\ https://hal.science/hal-01098822}

Submitted on 28 Jan 2015

HAL is a multi-disciplinary open access archive for the deposit and dissemination of scientific research documents, whether they are published or not. The documents may come from teaching and research institutions in France or abroad, or from public or private research centers.
L'archive ouverte pluridisciplinaire HAL, est destinée au dépôt et à la diffusion de documents scientifiques de niveau recherche, publiés ou non, émanant des établissements d'enseignement et de recherche français ou étrangers, des laboratoires publics ou privés. 


\title{
Cloud-based spectrum sensing system
}

\author{
first field results
}

\author{
Sylvain AZARIAN \\ Supélec / SONDRA \\ 3 rue Joliot-Curie - 91192 Gif-Sur-Yvette-France \\ sylvain.azarian@supelec.fr
}

\author{
Loig GODARD, Merouane DEBBAH \\ Supélec / Alcatel Lucent Chair on Flexible Radio \\ 3 rue Joliot-Curie - 91192 Gif-Sur-Yvette-France \\ loig.godard@supelec.fr - merouane.debbah@supelec.fr
}

\begin{abstract}
To estimate available spectrum for dynamic access in the context of cognitive radio, many of the designed systems rely on dedicated complex software defined radio (SDR) receivers with onboard powerful signal processing capabilities, connected through dedicated high speed networks to complex processing backends, transmitting their measurements in real time to a central system. Even if promising results have been achieved with these systems, we believe this architecture does not scale if an area wider than the lab has to be covered. In real conditions, one would have to select good installation sites, engineer antenna location and setup, install a high speed dedicated network link, falling back to the usual issues mobile phone carriers are facing, raising again the problems that were at the birth of small cell cognitive networks. Our approach was to look for solutions to deployment from the beginning of the study and integrate scalable capabilities with limited costs in the rationale of the architecture design.
\end{abstract}

Our 'Cloud SDR' architecture aims at being a Self-organized, dynamic, self calibrated, cloud oriented spectrum sensing system to perform continuous spectrum occupation measurements at a country-wide scale.

In this paper we describe the main features of the system and present preliminary results obtained with the sensing stations deployed in the suburbs of Paris, France, during the first semester of 2014.

Keywords- cognitive radio, dynamic spectrum access (DSA), spectrum sensing and estimation, experimental sensing setup

\section{INTRODUCTION}

To establish a reliable communication circuit between two radio nodes, knowledge of spectrum availability is required by both ends. This information can be estimated by the devices themselves, or provided by a dedicated sensing infrastructure doing a continuous spectrum survey on his side.

Relying on a sensing infrastructure makes the initial connection between the two nodes easier and faster, as they are given an initial common 'clear frequency', avoiding here the long steps to find suitable transmitting parameters (frequency and bandwidth). This sensing infrastructure is also viewed as a way for the communication regulators to open 'pay per use' or 'on demand spectrum access' models.

The design of a sensing infrastructure quickly raises the usual questions of the number of receiving nodes, the optimal location for these nodes and the balance between local and remote processing, leading to a complex system-level problem.
This work investigates the benefits of increasing significantly the number of receivers to estimate available spectrum for dynamic access. In particular, the case where:

- We limit the processing capabilities of each node to have low-cost receivers,

- We have no control of the receiver location and receiver availability: the receivers are of different types, different sensitivities, randomly placed, and can be off at any time,

- We do not use wideband antennas and calibrated front-ends.

We typically consider here the case of embedding a simple receiving hardware in an internet connected device like a cable or ADSL modem. They are shipped to their end-user who takes in charge the setup; we cannot make any assumption at design time of the final configuration, as we cannot make any assumption of the effective availability of the receiver.

The studied case here is different from the generally addressed context of networked or cloud-based cognitive radio systems designed for military applications like in ([1]): we focus on a country-wide class of system, running continuously to give the regulators tools to dynamically allocate portions of spectrum.

As the number of sensing nodes is supposed to be of much greater magnitude than the number of transmitting sources to be analyzed, we assume we'll have always more than one receiver per area of interest.

We will in the following present the studied configuration, outline the key elements of the designed architecture, highlight some aspects of the developed applications and algorithms, some results from the first measurement campaigns and finally propose the roadmap for the next main steps of the experiment.

As the paper is mainly oriented to present first field measurements, limited details on the underlying algorithms are presented here.

\section{STUDIED CONFIGURATION AND SIGNAL MODEL}

We make the following assumptions:

1. A set of $n_{t x}$ transmitters are transmitting unknown signals at unknown frequencies with unknown power, 
2. We can collect from $n_{r x}$ receivers placed at known locations $\left(n_{r x}>1\right)$ sequences of RF samples,

3. For a given region, the number of receivers cannot be predicted and varies with time,

4. The transfer function for each receiver is not known a priori, requiring a 'after deployment' calibration procedure.

5. $n_{r x}$ is much greater than $n_{t x}$, i.e. : $n_{r x} \gg>n_{t x}$.

A typical use case is described in Figure 1 where a set of 3 transmitters have to be estimated by using 70 available receivers randomly placed. Contour plots illustrates the decreasing signal power one receiver can expect at his location.

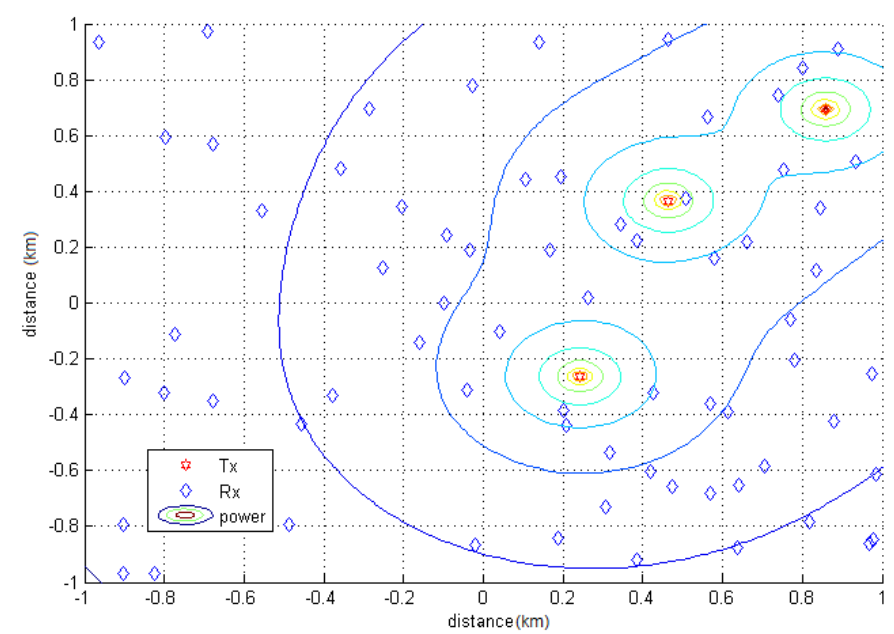

Figure 1- Typical scenario illustrated with 3 transmitters and 70 receivers.

At each receiver $R X_{i}$, we collect $n$ RF samples of an unknown number $p$ of complex exponentials in white noise:

$$
x_{i}(n)=\sum_{k=1}^{p} A_{k} e^{j n \omega_{k}}+w(n)
$$

It is assumed that the amplitudes $A_{k}$ are complex, $A_{k}=\left|A_{k}\right| e^{j \theta_{k}}$ with $\theta_{k}$ uncorrelated random variables that are uniformly distributed over the interval $[-\pi, \pi]$. The amplitudes and frequencies, $A_{k}$ and $\omega_{k}$, are not random but unknown; and $w(n)$ being the white noise contribution.

$A_{k}$ is related to the signal original power affected by attenuation from considered transmitting source to the considered receiver, this attenuation being the cumulative effect of the antenna plus RF front-end response and propagation effects. Each transmitted signal can be composed of more than one single complex sinusoid; hence the received signal is the contribution of all the complex sinusoids from all transmitters. The $p$ complex exponentials at the receiver can be written as (neglecting multipath or echoed delayed signals): $\sum_{k=1}^{p} A_{k} e^{j n \omega_{k}}=\sum_{k=1}^{p T X 1} A_{1, k} e^{j n \omega_{1, k}}+\ldots+\sum_{k=1}^{p T X . .} A_{M, k} e^{j n \omega_{M, k}}$

Where $A_{j, k} e^{j n \omega_{j, k}}$ is one complex exponential coming from transmitter j, with amplitude $A_{j, k}$ and frequency $\omega_{j, k}$.

Many algorithms have been designed to extract the complex exponentials from received signal $x_{i}(n)$. These methods ([2], [3], [4]) process finite sample sequences and try to estimate the number of complex exponentials and their respective powers. We focus here on methods relying on eigendecomposition of the autocorrelation sequences $R_{i}$ of the received signal, building the signal and noise subspaces. An interesting property of such a decomposition is the relation between the number of complex exponentials and the dimension of the signal subspace. Such a decomposition on the data collected from different sensors does not help to estimate which of the identified carriers come from the same transmitter (i.e. divide signal space into transmitter space);

Considering two different receivers $R X_{i}$ and $R X_{j}$, each collecting simultaneously RF samples $x_{i}(n)$ and $x_{j}(n)$, we have (neglecting again multipath or echoed delayed signals) the following hypothesis:

1. Two receivers sufficiently close to each other will receive strongly correlated $x_{i}(n)$ and $x_{j}(n)$ sequences,

2. Two receivers moderately separated will have strong correlations between $x_{i}(n)$ and $x_{j}(n)$ only in some sub-bands (only a subset of the complex exponentials is common)

Our target goals are to:

1. Design a remote, field-based, calibration procedure to estimate the front-end plus antenna response,

2. Estimate the number of transmitters;

3. Estimate the transmitters location;

4. Estimate the bandwidth each transmitter uses and the related spatial power distribution.

For this purpose, we study the following method:

1. Collect sets of data at the same frequency simultaneously from receivers in the same region;

2. Perform spectrum estimation from these sets to split received bands in 'occupied sub-bands'

3. Perform cross-correlations in same sub-bands between receiver pairs to check that the same signal was received.

At this point, we can estimate the probability of having received the same signal over a subset of receivers and decide if there was a transmitter active at the time of observation. This 
operation needs then to be repeated over time to estimate the type of transmission (continuous, intermittent, etc.).

As this paper is focused on some first field results, these points will not be covered here.

\section{SYSTEM ARCHITECTURE OVERVIEW}

\section{A. A set of randomly placed devices}

First, we remind here that we make no choice on the location of the sensing node and make no assumption of the quality of the antenna connected to the receiver used, assuming we will have sufficient number of sensing nodes to be able to select the most appropriate one, depending on measurement requirements, after remote calibration sequence.

The targeted device can be an add-on embedded for example inside a DSL/cable internet modem with embedded or external antenna, as depicted in figure 2.

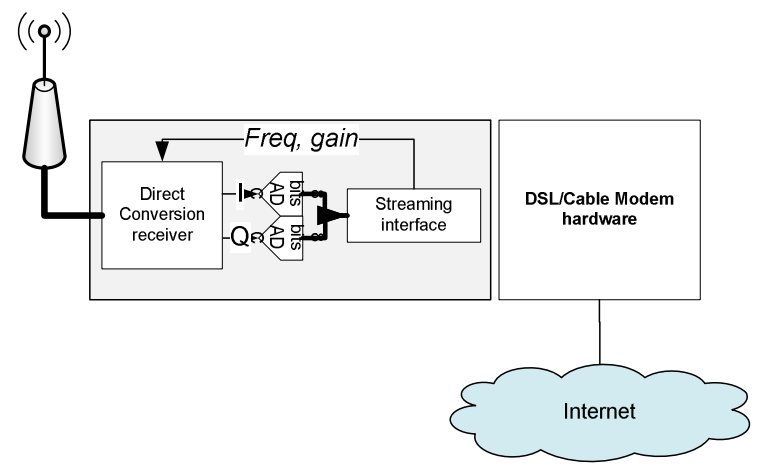

\section{Figure 2 - SDR receiver as an add-on inside DSL/Cable} modem.

Typically this can be a direct-conversion software defined receiver, running an embedded operating system like Linux. For example, if a significant fraction of Internet boxes was fitted with this type of add-on, this would lead to an important number of sensing nodes per square kilometre.

As such a device would probably not be installed outdoors; GPS should not be used to estimate the device location. To estimate sensor's position, a geocoding scheme is used, transforming the shipping address of the device to its latitude and longitude. This gives good accuracy, transforming internet connection IP address to coordinates.

Each device contains a unique identifying ID, paired in the system database with targeted address coordinates. A specific algorithm is required to check location consistency, assuming irrelevant addresses have limited probability. This point is out of the scope of this paper.

Once started, the sensing node software performs a DNS lookup to retrieve an available registering remote server. It then starts an authentication procedure, using its assigned unique ID. Once identified, the device is registered in a geographic database. First appearing devices are listed to enter a calibration procedure.

Because the internet connection is not reliable, a ping/pong procedure is then initiated between nodes and servers to maintain the node availability list accurate. When network connection dies, the registration procedure is tried again. On the directory server side, any node with no activity for a while is deleted.

\section{B. A set of Internet connected machines}

In the previous section, we briefly described the sensing node and registration system. As described by figure 3, other types of nodes are involved in the system.

Orchestration nodes manage the direct connection to the sensing nodes. They receive the RF samples and forward them to the requester, namely the processing nodes or client nodes. For scalability requirements, the number of receivers connected to one single orchestration depends on the current system load. Typically, sensing nodes can be moved from one server to another between two connections.

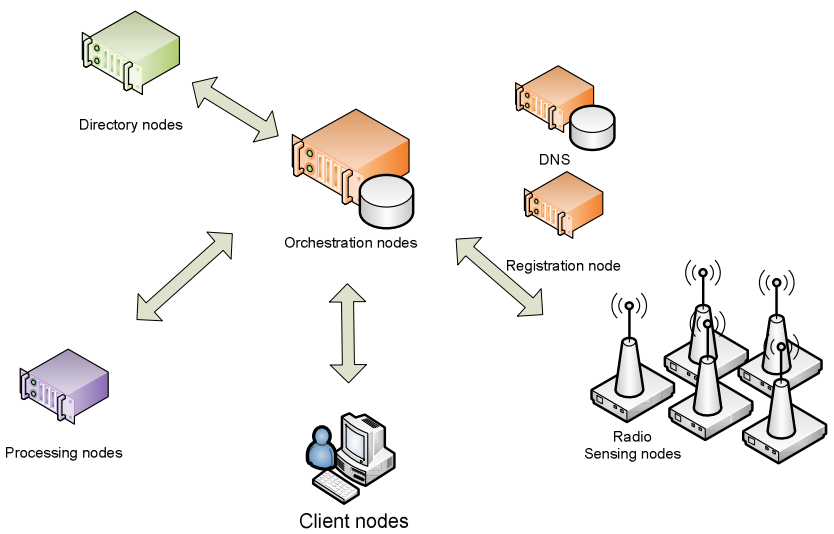

Figure 3 - Hierarchical view of nodes.

The geographical database is here to give a list of available nodes for a given region.

\section{Message passing and communication between nodes}

As the number of effective nodes does not remain constant over time, connection from clients to sensing nodes is done by message relaying through the orchestration node, managing at his side the connections/disconnections of nodes. Any request issued by a node is transmitted asynchronously depending on requested application. A typical "sensing request" sequence is shown in figure 4.

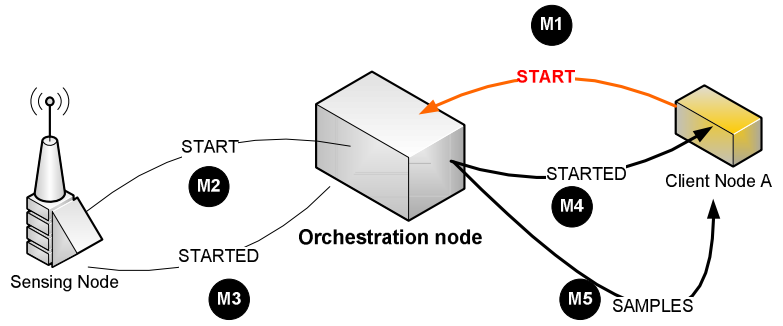

Figure 4 - Asynchronous message passing.

Client node $A$ issues a 'start request' to one orchestration node (message M1). This request is forwarded to the target node (message M2). When the sensing node has started the requested operation, an acknowledgment message is sent back 
(message M3). This message is finally routed to the client (message M4). Meanwhile the first samples are starting to flow from the sensing node and routed to requester (message M5).

Messages are routed between nodes using priority queues, with highest priority set to status updates and acknowledgments. Doing so, a requester node receives the confirmation that an operation has been successfully started before receiving the processing results.

\section{SENSING EXPERIMENT AND RESULTS}

Doing realistic simulations of such type of architecture is quite difficult, as estimating realistic propagation models and noise distributions are research topics by themselves.

Another point often neglected is related to the problem of having time or frequency synchronization between the different observers. As a continuation of pre-existing works on SDR platforms, it was been decided to perform a 'spectrum sensing' experiment, aiming at:

1. Testing the architecture in real conditions, focusing on using Internet as a communication channel between system and sensors,

2. Testing a variety of different antenna and receiver locations which were not planned (or engineered),

3. Estimating the influence of non perfect frequency synchronization, non perfect sampling rate, and non perfect time synchronization.

The following situations were to be tested:

- Perform automatic calibration/sensor validation;

- Explore FM broadcast band and test system capacity to identify transmitters, with 'ground truth' available. We would have here several receivers with same signals for the most powerful transmitters;

- Explore higher frequencies, like ISM or amateur bands, where the coverage is much more limited and signal would be received by one or maximum two stations.

\section{A. Sensing devices location}

For the experiment, a call for volunteering was sent to French licensed Amateur Radio operators [5] and was sent to radio hobbyist willing to install a custom application in their own computer, connected to the internet. A low-cost, low quality DVB-T USB dongle receiver [6] was provided. This device embeds a direct conversion tuner and two 8 bits ADCs, with sample rates up to $3 \mathrm{MSps}$. This choice to use skilled volunteers was driven by the fact that this solution would not raise technical difficulties for antenna installation, amateurs having their own antenna setup.

The exact number of participants cannot be disclosed, but figure 5 shows a subset of active sensing stations for one of the sensing trials. These stations are distributed over Paris, France. The most distant station (tag 12) was about 45
Km away from the Eiffel Tower (tag TE). Total area covered by sensors is around $90 \times 60 \mathrm{~km}$.

Different types of configurations where tested: Linux machines, Windows XP notebooks, Raspberry PI systems. Four Raspberry Pi sensing nodes were used for the experiment. During the different phases of the experiment, conducted over February to May 2014, the volunteers were asked to turn their local system on, depending on their availability.

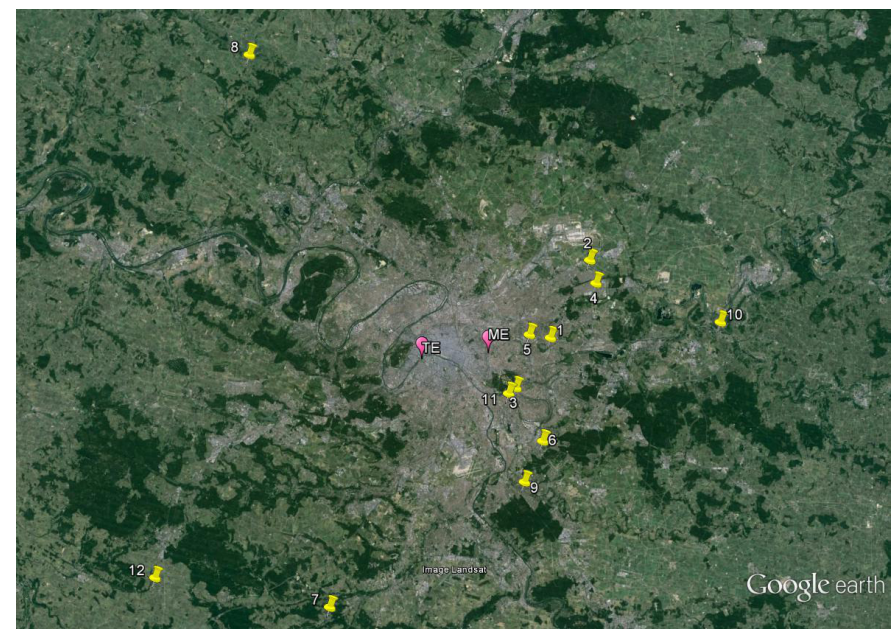

Figure 5 - Area of the experiment: Paris and suburbs

To manage communication with volunteers, a dedicated web site was used to deliver software releases, installation tutorials, and experiment planning news.

\section{B. Signal differences, synchronization - examples}

During the campaigns, a subset of the available receivers were selected periodically and asked to perform acquisition sequences as follows:

1. Frequency requested in the following ranges: [88$108 \mathrm{MHZ}]$; [144-146 MHz];[430-440 MHz]; [866-870MHz];

2. 512000 samples acquired at 1 MSPS were asked at each observation;

As an example, we compare in the following the signals received simultaneously from two very close receivers, tagged 2 and 4 in figure (distance is $3 \mathrm{Km}$ ). Both spectrograms show very comparable signals at $-0.1 \mathrm{MHz}$ and $+0.3 \mathrm{MHz}(90.9$ and 91.3 MHz), but we can hardly see signal at $-0.4 \mathrm{MHz}$ on second plot. To estimate the time synchronization between these two signals, a band pass filter centered at $0.3 \mathrm{MHz}$ and cross-correlation test was performed, showing on the second plot a delay of 9610 samples (9.610 milliseconds). Figure 8 shows cross-correlation after time correction.

The two spectrograms in figure 7 compare the signal evolution around $91 \mathrm{MHz}$, over 500 milliseconds. 


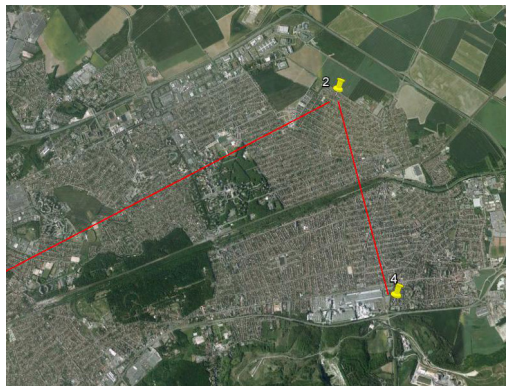

Figure 6 - Zoom around RX2 and RX4
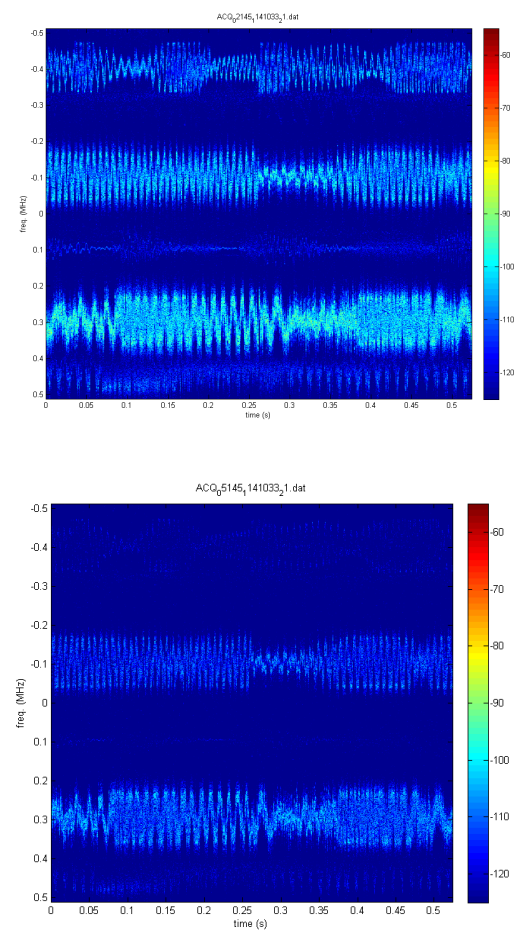

Figure 7 - Spectrograms from simultaneous observations

Evaluation of the average delay has to be conducted over the different trials to estimate validity of implemented technique to distribute requests over sets of receivers.

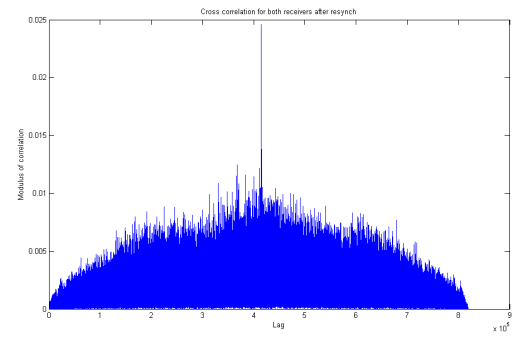

Figure 8 - Cross correlation after delay correction

\section{CONCLUSION}

The architecture of a distributed, cloud oriented, spectrum sensing system was designed, implemented and deployed to validate key concepts. In this work we tried to estimate the potential outcome of using a 'massively distributed' set of randomly installed Software Defined Receivers, connected to non-planned antennas or dedicated TCP/IP networks.

Several tens of sensing stations were installed in the first semester of 2014 around Paris and after individual test procedures, sensing campaigns were conducted. At the time of writing only preliminary conclusions can be drawn from the tens of giga samples collected, but we have successfully validated:

1. The capacity of the designed architecture to cope with a dynamic set of sensors,

2. The capacity of the system to distribute processing over different nodes to perform signal processing or database management;

The following important points are still under evaluation at this time, lacking detailed analysis:

1. Distribution of the delays when simultaneous acquisitions are requested over a wide range of sensors;

2. Influence of the different TCP/IP speed available at each sensing station ;

3. Influence of the different receiving frequencies and sampling rates.

\section{ACKNOWLEDGMENT}

The authors would like to thank all the volunteer amateurs involved in the project and also all the people who helped to manage the setup over the tens of receiving stations. In particular, we would like to thank:

- The REF, the French National amateur radio organization (http://www.r-e-f.org/);

- Among the many participants the set of radio amateurs who lent their antennas, computers, Internet connections for the experience.

\section{REFERENCES}

[1] F. Ge, H. Lin, A. Khajeh, C.J. Chiang, A.M. Eltawil, C.W. Bostian, W. Feng, R. Chadha, "Cognitive Radio Rides on the Cloud", The 2010 Military Communications Conference.

[2] V.F. Pisarenko, "The retrieval of harmonics from a covariance function," Geophysics, J. Roy Astron Soc., vol 33, 1973.

[3] R. Schmidt, "Multiple Emitter location and signal parameter estimation", Proc. RADC Spectrum Estimation Workshop, 1979,

[4] M. Wax and T. Kailath, "Detection of signals by information theoretic criteria", IEEE Trans. Acoust., Speech, Sig. Proc., vol. ASSP-33, 1985,

[5] What is amateur radio ? http://en.wikipedia.org/wiki/Amateur_radio, as per may 2014.

[6] Osmocom SDR - http://sdr.osmocom.org/trac/wiki/rtl-sdr 